\title{
Activités
}

14-1 | 2017

Varia

\section{Construire et conduire une intervention en ergonomie}

Questions pour la formation professionnelle

Building and driving an intervention in ergonomics: questions on professional training

Viviane Folcher, Anne Bationo-Tillon et Stella Duvenci-Langa

\section{OpenEdition}

Journals

Édition électronique

URL : https://journals.openedition.org/activites/2956

DOI : 10.4000/activites.2956

ISSN : $1765-2723$

Éditeur

ARPACT - Association Recherches et Pratiques sur les ACTivités

Référence électronique

Viviane Folcher, Anne Bationo-Tillon et Stella Duvenci-Langa, « Construire et conduire une intervention en ergonomie », Activités [En ligne], 14-1 | 2017, mis en ligne le 15 avril 2017, consulté le 21 septembre 2021. URL : http://journals.openedition.org/activites/2956 ; DOI : https://doi.org/10.4000/activites. 2956

Ce document a été généré automatiquement le 21 septembre 2021.

\section{(c) (1) $\$ \Theta$}

Activités est mis à disposition selon les termes de la licence Creative Commons Attribution - Pas d'Utilisation Commerciale - Pas de Modification 4.0 International 


\title{
Construire et conduire une intervention en ergonomie
}

\author{
Questions pour la formation professionnelle \\ Building and driving an intervention in ergonomics: questions on professional \\ training
}

Viviane Folcher, Anne Bationo-Tillon et Stella Duvenci-Langa

\section{NOTE DE L'ÉDITEUR}

Article soumis le 5 janvier 2016, accepté le 4 novembre 2016

\section{Introduction}

1 La construction de ressources pour l'agir ergonomique est un enjeu de la formation au métier et nous souhaitons dans cet article rendre compte d'un dispositif de formation professionnelle en ergonomie, en expliciter les fondements et en décrire la mise en œuvre.

2 Notre contribution se situe à la croisée de formats habituellement distincts que sont le retour d'expérience et l'écriture scientifique. Retour réflexif qui vise le partage de notre pratique, ce texte met en mots ce qui se déroule à l'occasion du séminaire de suivi de stage d'apprentis ergonomes en Master. Il prend appui sur un ensemble diversifié de traces, observations ouvertes, notes manuscrites et entretiens menés auprès d'étudiants diplômés dont nous détaillerons la méthodologie par la suite. Nourri d'un ensemble de contributions scientifiques, ce dispositif est fondé sur l'autonomie des étudiants sur les terrains de leurs premières interventions. Il met au premier plan la dynamique individuelle et collective des apprentissages des étudiants, et met en lumière la coactivité encadrants-étudiants. 
3 À distance d'un modèle à dominante épistémique d'un étudiant récepteur de concepts et de méthodes à mettre en application, la formation que nous animons se situe dans une perspective développementale d'un sujet historique et culturel, bénéficiant d'un ensemble d'acquis sociaux et culturels qu'il s'approprie et renouvelle dans la diversité de ses milieux d'activité et de vie (Vygostki 1985). Ainsi milieux et sujets ne sont pas superposables mais au contraire s'engendrent réciproquement. Ils sont saisis et appropriés par un sujet actif, capable de s'y inscrire, d'en identifier les limites, d'observer les effets de ses expériences, de les transformer et d'être transformé par eux (Wallon 1985). La formation professionnelle est un de ces milieux, espace de socialisation et de développement professionnel autant que personnel. Elle prend appui sur une définition précise du sujet apprenant, acteur de son apprentissage, riche de ses expériences et auteur de son devenir.

Dans le champ de la formation des adultes, le terme d'apprenance propose une vision de l'adulte en formation qui est proche de nos propres travaux. Considérer l'adulte comme sujet proactif aux intentions de formation c'est, en accord avec P. Carré (2005), accepter de renverser la perspective selon laquelle l'apprentissage serait le résultat de la (seule) intervention sur la personne formée. Dans une stimulante contribution, l'auteur explore les racines historiques et scientifiques d'un paradigme, que l'on pourrait qualifier d'extrinsèque, qui perdure en dépit des nombreux travaux ayant montré son caractère peu opérant (Carré, 2016). À partir du constat d'une erreur pédagogique fondamentale, l'apprenance propose une conceptualisation de l'apprentissage et du développement des compétences dans un tripode qui comprend:

- Les dispositions à apprendre c'est-à-dire les expériences mises en patrimoine. Elles renvoient d'une part aux déterminants internes identifiés en ergonomie et, d'autre part, au concept de capacité et de pouvoir d'agir sur lequel nous reviendrons en détail dans ce texte ;

- Les pratiques apprenantes c'est-à-dire l'ensemble des activités d'apprentissage et des pratiques réflexives déployées par les sujets en situations. La visée est compréhensive, centrée sur les dynamiques d'apprentissage des sujets, proche en cela des travaux en psychologie historico-culturelle intéressés par les processus de développement des personnes dans l'unité de leurs vies auxquels nous nous référons ;

- Les situations d'apprentissage c'est-à-dire l'ensemble des situations formelles et semiformelles. Les trois niveaux de situations d'apprentissage qui sont envisagés - situationnel, contextuel et global - entrent en écho avec les propositions de classes de situation, famille d'activité et domaine d'activité qui ont été proposés en ergonomie.

5 Notre contribution traite de la mise en œuvre d'un dispositif de formation en prenant la double focale des activités apprenantes des étudiants praticiens et des encadrants à l'université. Elle rencontre les préoccupations d'un ensemble de travaux intéressés par l'interaction entre dispositions des sujets apprenants et dispositifs de formation (Lameul, Jezegou, \& Trollat, 2009). Dans cet ouvrage collectif, une grande diversité de situations de formation adressées à une variété de sujets apprenants a été analysée. Nous retiendrons des résultats reportés quelques points essentiels. L'importance d'un positionnement épistémologique du sujet apprenant tout d'abord, il sera entendu dans les termes d'une construction continuée de soi-même dans l'interaction avec un dispositif de formation. En second point, les travaux convergent vers la reconnaissance que l'efficacité d'un dispositif de formation repose, notamment, sur sa capacité à accueillir les dispositions des apprenants dans leur diversité. Enfin, troisième point, la formation en tant que processus et en tant que dispositif y est définie comme un lieu de relations, d'interactions, de 
tensions et de transactions qui se tissent entre le dispositif - son cadre et ses acteurs - et les dispositions des apprenants.

Le sens de notre propos est de donner à voir la nature de ces interactions, tensions et transactions qui se déploient à l'occasion d'un séminaire de suivi de stages professionnels entre des encadrants universitaires et des jeunes praticiens en formation.

7 En première partie du texte nous exposons les cinq partis pris conceptuels et méthodologiques qui structurent le séminaire de suivi de stage. En seconde partie nous détaillons le dispositif de formation en explicitant son principal objectif, former par la mise en débat des interventions en train de se faire. Nous rendons compte ensuite des moments clés qui rythment l'accompagnement et mettons en lumière les tensions, relâchements et leurs dénouements. Puis nous éclairerons la co-activité enseignants et étudiants afin d'en dégager les invariants et les ressources mobilisés par les uns et les autres.

8 En troisième partie nous menons une discussion sur les thèmes de l'apprentissage et des dispositifs de formation professionnelle.

\section{Partis pris de formation}

\subsection{Des praticiens capables d'ergonomie}

L'encadrement universitaire considère les étudiants, jeunes ergonomes, dans les termes de sujets capables au sens de l'approche proposée par Rabardel (2005). Sujet pragmatique $\mathrm{du}$ «je peux» avant que d'être un sujet épistémique du «je sais», le sujet capable est un sujet de l'agir qui œuvre dans le monde, cherche à faire advenir des transformations, vise l'atteinte de résultats : il est un sujet en activité dans sa dimension productive. Sujet en devenir et auteur de son développement, il s'approprie ce qui est donné, disponible ou prévu, construit des ressources et y associe un ensemble de valeurs, configure ses situations d'activité pour le futur: il est un sujet en devenir dans sa dimension constructive (Rabardel \& Samurçay, 2001). Une définition en extension du pouvoir d'agir a été proposée à la suite de ces travaux couvrant l'ensemble des dimensions de l'action sensée et normée (Folcher \& Rabardel, 2004 a, b). La distinction entre capacité et pouvoir d'agir a ensuite été précisée (Rabardel op.cit), des propositions méthodologiques d'objectivation des capacités et des pouvoirs d'agir ont pu voir le jour (Gouédard \& Rabardel, 2012) et un ensemble de travaux a documenté la dimension collective du pouvoir d'agir au sein des organisations (Poret, 2015 ; Poret, Folcher, Motté, \& Haradji, 2016).

10 Le parti pris du sujet capable en ergonomie prend sa source dans les perspectives historiques et culturelles de psychologie dont nous venons de parcourir quelques contributions. Il n'est pas sans lien avec les propositions dans le champ de la pédagogie des adultes, en particulier les travaux sur l'apprenance que nous avons évoqués précédemment. Accorder le primat au sujet capable et outiller son pouvoir d'agir en situation d'intervention, c'est donner la priorité à la logique d'un sujet apprenant, proactif et auteur de son développement à l'occasion d'une «formation qui, pour être effective, requiert un partenaire actif d'un contrat avec soi-même et/ou avec son contexte de travail et de vie, disposé à entreprendre les activités, de brève ou de longue durée, requises en vue de l'atteinte des objectifs » (Carré op. cit, p. 6-7). 
11 Ce parti pris est également nourri des orientations initiales de formation à l'ergonomie développées au CNAM dans les années 70 sous l'impulsion d'A. Wisner, en particulier l'enseignement de TPB (Dessors, 2006 ; Wisner, 1990) qu'il reprend à son compte et poursuit. Conçu comme une formation à la pratique ergonomique et à l'analyse du travail, cet enseignement s'appuyait sur un collectif d'enseignants en position d'accompagner et de guider les interventions des apprentis ergonomes. Le projet visait des objectifs de formation professionnelle et de développement d'une connaissance puis d'une recherche sur la pratique (Daniellou, 2006 ; Garrigou, 1992).

Nous aurons l'occasion dans la suite de ce texte de revenir sur le gisement constitué par l'école francophone d'ergonomie fondée au CNAM et nous allons à présent envisager notre second parti pris de formation.

\subsection{Intervenir dans les milieux de travail et de vie}

13 Qu'il s'agisse de situations de la vie quotidienne ou de travail, la démarche d'intervention ergonomique conserve ses principales caractéristiques. C'est notre second parti pris de formation. De nombreux travaux ont souligné très tôt l'importance de penser les milieux de travail et de vie de manière holistique.

En 1969, lors du $4^{\mathrm{e}}$ congrès de la S.E.L.F, l'ergonomie y est définie comme «l'étude scientifique de la relation entre l'homme et ses moyens, méthodes et milieux de travail. Son objectif est d'élaborer avec le concours de diverses disciplines qui la composent un corps de connaissances qui, dans une perspective d'application, doit aboutir à une meilleure adaptation à l'homme des moyens technologiques de production et des milieux de travail et de vie ». En ergonomie, l'analyse de l'asservissement réciproque du travail sur la vie a été un angle d'étude dès les années 70. L'impact du travail en horaires décalés sur les rythmes biologiques a été ensuite largement documenté (Barthe, Quéinnec, \& Verdier, 2004 ; Gadbois, 1975 ; Gadbois \& Quéinnec, 1984 ; Prunier-Poulmaire 1997).

En psychologie, un ensemble de travaux envisagent l'existence humaine dans une perspective où les milieux, institutions et organisations constituent les conditions et les obstacles à la construction de la personne (Malrieu, 1979; Wallon op.cit). Ils ouvrent un espace de structuration réciproque dans lequel « restructuration organisationnelle et restructuration de soi se répondent donc, même si ces processus d'inter-structuration s'effectuent selon des formes et des rythmes différents » (Curie, 2000 p. 24). Dans la ligne des préoccupations de P. Malrieu, J. Curie et V. Hajjar centrent leurs travaux de recherches sur l'interdépendance des conduites de la vie quotidienne, interrogent « la vie en temps partagé » (Curie \& Hajjar, 1987) assumant que nos activités forment un système aux ressources finies dans lequel chaque activité génère des contraintes pour le développement des autres. Dans les années 90, les travaux d'Y. Clot, en clinique de l'activité, ont invité à penser une psychologie des milieux de travail et de vie (Clot, 1995).

L'ensemble de ces contributions invite à dépasser la dichotomie acquise entre le travail et la vie quotidienne et à progresser dans une vision de l'homme dans l'unité de sa vie et la continuité de ses activités (Folcher, 2010). De nouveaux terrains d'investigation peu explorés jusque-là s'ouvrent : usages de mobiliers de jardins en lien avec les équipes de conception (Couillaud, 2011), usages de pompes à insulines par les patients diabétiques en lien avec les associations de patients (Guenfoud, 2015), analyse des activités domestiques de clients producteurs d'énergie photovoltaïques et recommandations pour la conception 
(Lahoual, 2012), nouvelles technologies au service de l'activité muséale (Bationo-Tillon, Lahoual, Alexandre, Mateev, Tichadou, \& Decortis, 2013).

Examinons maintenant notre troisième parti pris de formation.

\subsection{De la demande à l'offre, le champ des possibles de transformation}

Tendue entre une demande et un champ de transformations possibles, l'intervention en ergonomie vise à transformer les situations de travail, et plus largement l'ensemble des situations d'activité humaine, selon des critères de santé et d'efficacité (Durrafourg, 2004 ; Guérin, Laville, Daniellou, Duraffourg, \& Kerguelen, 2001 ; Laville \& Volkoff, 2004). Elle tient ensemble l'ambition de comprendre et celle de transformer et implique, de ce fait, une démarche de co-construction avec une diversité d'acteurs, dans un contexte donné, à un moment donné. En ce sens toute intervention est la conduite d'un projet (Saint-Vincent, Vezina, Bellemare, Denys, Ledoux, \& Imbeau, 2011). Cela constitue notre troisième parti pris de formation.

Point de départ fréquent d'une intervention, la demande est toujours l'expression plurielle de problèmes, de préoccupations, d'attentes qui sont inscrites au cœur de liens et d'histoires entre des personnes (Martin, Daniellou, \& Escouteloup, 1996). Précisément formulée au départ, elle peut se réouvrir en une diversité de questions et de préoccupations. Faiblement formulée, voire absente, elle peut émerger dans la rencontre avec les acteurs.

20 Implicites ou explicites les expressions d'une demande doivent faire l'objet d'une exploration approfondie pour en saisir le processus d'élaboration, en comprendre les rapports de force et construire les espaces possibles pour l'action (Durrafourg, 2004).

21 Afin de « reposer avec les acteurs de l'entreprise les questions que doit traiter le projet dont il est question dans une approche systémique et non plus en termes de solution point par point» (Noulin, 2002 p. 143) deux outils d'analyse sociale et causale de la demande ont été développés (Rabardel, Folcher, \& Le Jollif, 1996). Ils visent à structurer la compréhension de la situation au plan des acteurs, de leurs enjeux et des problèmes exprimés - il s'agit de l'analyse sociale - et au plan du travail dans ses déterminants et ses effets sur les personnes et les situations - il s'agit de l'analyse causale -.

L'outil d'analyse sociale distingue les acteurs exprimant un problème, les personnes susceptibles d'être concernées par le problème ainsi que les éléments de contexte. La figure 1 en propose une illustration. 
Figure 1 : Outil d'analyse sociale de la demande.

Figure 1: Tool for social demand analysis

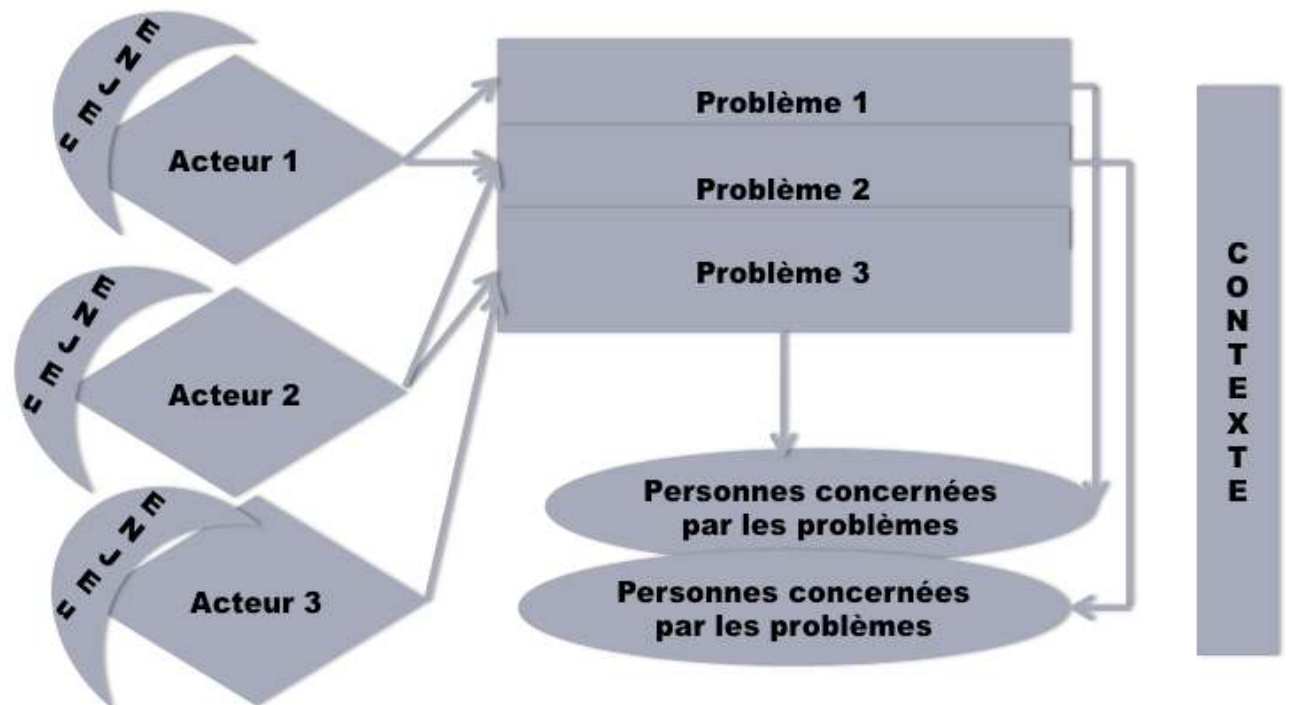

23 L'analyse sociale reporte les dires des acteurs au plus près de leurs expressions singulières : ils sont constitués en problèmes, préoccupations ou attentes. Les enjeux sont progressivement déduits à partir des problèmes exprimés. Les personnes potentiellement concernées par les problèmes sont identifiées. Par cette première analyse, la diversité des problèmes est documentée, les lignes de tension et de contradictions possibles apparaissent et la dimension historique des problèmes se dévoile. Pas à pas, les espaces possibles pour l'action se dégagent.

En complément, l'outil d'analyse causale vient étayer l'analyse sociale par une compréhension du travail dans ses déterminants et ses effets et l'identification des solutions envisagées et/ou mises en place. La figure 2 en propose une illustration. 
Figure 2 : Outil d'analyse causale de la demande. Figure 2: Tool for causal demand analysis

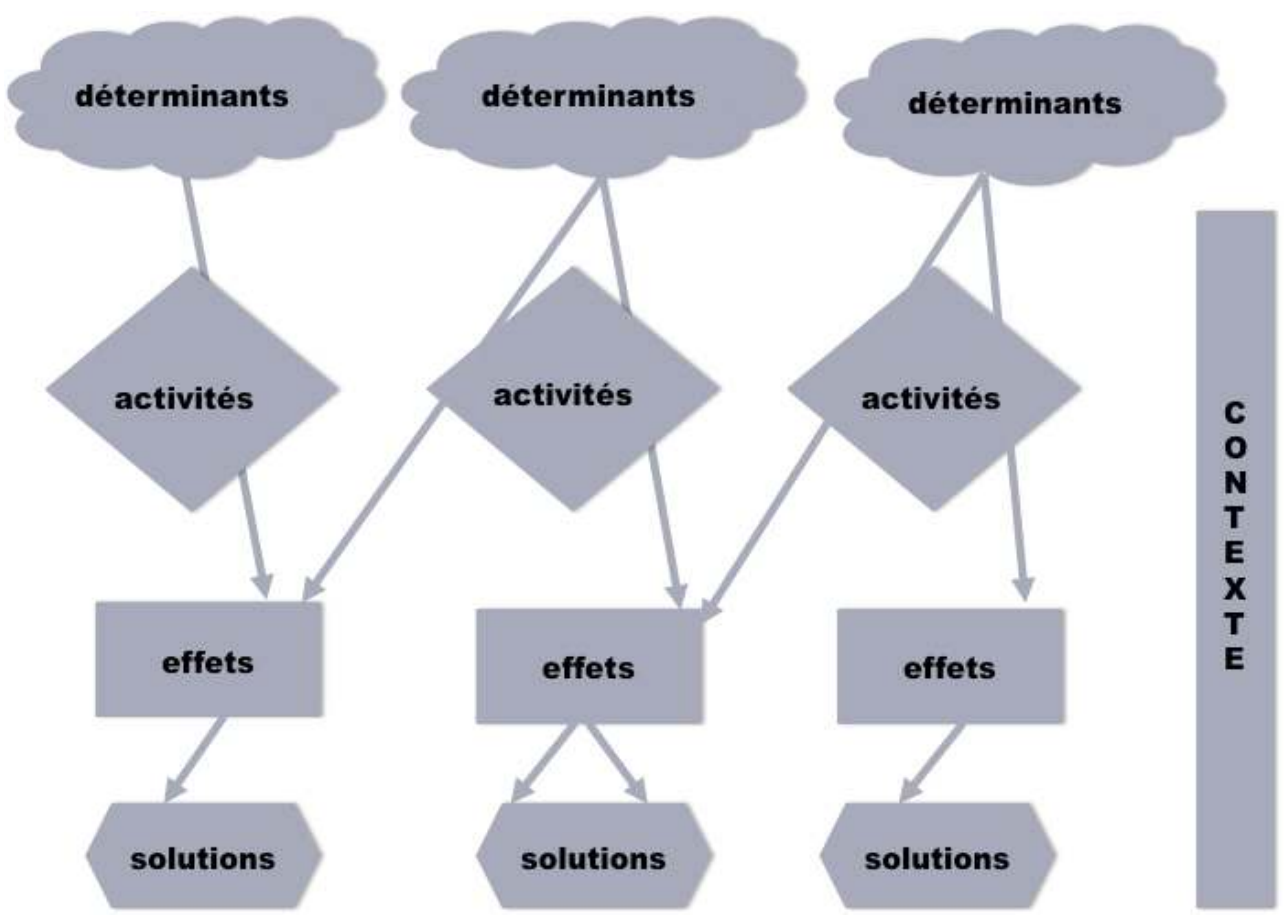

Écoute et report des dires des acteurs au plus près de leurs expressions singulières sont à observer, tout comme dans l'analyse sociale. Une première compréhension des situations de travail se construit progressivement. Elle est globale et schématique et vise à établir une cartographie des situations qui vient enrichir l'analyse sociale et permet de l'ancrer dans le travail et son histoire, en particulier par une connaissance des solutions. Elle contribue à orienter les analyses du travail qui seront réalisées par la suite : choix des situations, choix des services, choix des postes de travail...

Analyse sociale et causale de la demande étayent la proposition d'intervention que le praticien formulera. Cette proposition recevra l'aval des acteurs qui sont parties prenantes de l'intervention pour que celle-ci débute ${ }^{1}$. Pour autant, l'analyse n'est pas figée et pourra connaître des enrichissements et des ajustements au fur et à mesure de l'avancée du travail.

Ces deux outils permettent l'immersion et la distanciation. L'immersion par la collecte riche d'éléments hétérogènes (documentations, notes, entretiens informels, rencontres, photos...) qui permet de construire une vision multidimensionnelle du réel à partir d'une appréhension de son caractère foisonnant, divers, variable, contradictoire... Distanciation par une objectivation minutieuse des faits, qui produit un éloignement du caractère foisonnant du réel pour lequel l'immersion a été d'abord nécessaire.

Ils favorisent un double apprentissage du regard porté sur le travail. Résolument myope, pour reprendre une formule chère à $\mathrm{M}$. de Montmollin, dans le recueil au plus fin du travail « tel qu'il se vit et tel qu'il se dit », le regard construit une vision panoramique qui vient embrasser la globalité sans évacuer la singularité. L'intervenant intègre progressivement l'immersion et la distanciation et développe une capacité à circuler entre ces deux pôles. 

déterminé s'élabore à cette occasion, et si les étudiants envisagent souvent l'analyse de la demande comme une étape à surmonter, ils découvrent petit à petit son caractère continu et les bénéfices associés.

bilisé initialement au service de la formation à l'intervention, cet outillage a été transféré dans une pratique de conduite de projet où il structure la phase initiale de la démarche de conception pour et dans l'usage proposée, à savoir l'analyse de la situationprojet (Folcher, 2015 ; Folcher, Zreik, Ben Rajeb, \& Leclercq, 2013).

\subsection{Acteurs et contributions, construire et conduire l'intervention}

31 L'intervention est une démarche transformative fondée sur la compréhension du travail réel, l'analyse de l'activité en est une spécificité ${ }^{2}$ Ancrer les transformations dans l'activité est le quatrième parti pris de la formation. Il reconnaît que le seul diagnostic des mauvaises conditions de travail ne peut suffire à transformer effectivement le travail. Ce constat a conduit la discipline à interroger sa contribution effective à la conception. Sur le plan international, les travaux en matière de conception participative (Bjerknes, Ehn, \& Kyng, 1987 ; Ehn, 1988) ont ouvert la voie à la conduite de projet fondée sur le travail réel cependant que dans le périmètre francophone, l'identification du paradoxe de l'ergonomie de conception (Theureau \& Pinsky, 1984) a donné lieu à un ensemble de propositions pour conduire les projets en référence à l'activité future (Barcellini, Van Belleghem, \& Daniellou, 2013 ; Daniellou, 2004 ; Garrigou, Thibault, Jackson, \& Mascia, 2001; Martin, 2004), articuler des mondes professionnels et construire un monde commun (Béguin, 2010), ou encore concevoir pour et dans l'usage (Folcher, op. cit).

L'ensemble de ces contributions scientifiques ouvre des perspectives en matière de formation. Penser l'intervention dans les termes d'une conduite de projet (Saint-Vincent et al., op. cit) invite à considérer un double objectif de formation professionnelle. Il s'agit de construire et de conduire l'action ergonomique :

- Construire renvoie à la mise en œuvre de la démarche d'investigation propre à l'ergonomie qui partage avec d'autres disciplines d'intervention certaines méthodes, tout en conservant la spécificité d'être centrée sur l'analyse du travail et de l'activité, des premières observations au pré-diagnostic, du diagnostic à la construction de pistes de transformation ;

- Conduire a trait aux relations qu'il importe de nouer avec les acteurs et aux modalités de coconstruction de l'intervention qu'il faut instaurer dans le temps de la mission et au-delà, en considérant que l'enjeu de l'action ergonomique est de créer une dynamique durable de transformation permettant de concevoir des artefacts et des organisations au service du travail humain.

Le dispositif pédagogique de suivi de stage vise à outiller les ergonomes praticiens pour construire et conduire leurs interventions. Aux outils d'analyse sociale et causale de la demande s'ajoutent les groupes de suivi dans l'entreprise. Les étudiants sont invités à les mettre en place à partir de la phase de reformulation de la demande. Diversement nommés en fonction des structures déjà existantes et des pratiques en vigueur, les groupes de travail sont, en miroir du suivi de stage universitaire, des espaces potentiels de mise en débat des interventions dans les entreprises. 


\subsection{Problèmes de société, questions de science}

Construction et conduite de l'intervention ne peuvent exister sans capitalisation des acquis qui contribuent à la solidité de la discipline et en permettent son renouvellement. Ceci est notre cinquième parti pris.

Plaçons-nous depuis une perspective académique. Depuis 2007, les directives ministérielles invitent les universités à intégrer au sein des formations de Master les dimensions recherche et professionnelle quand elles étaient disjointes jusque-là. Force est de constater qu'une séparation entre la recherche et l'intervention perdure de nos jours. Or cette évolution structurelle qui touche les institutions invite la communauté à progresser sur la question de leur articulation. Pour mettre au travail la question, envisageons la proposition suivante:

«Il n'est pas de pratique de recherche sans capacité à mener une intervention ergonomique, il n'est pas de pratique d'intervention sans un ancrage dans les travaux réalisés, diffusés et capitalisés au sein de la discipline ».

L'intervention en ergonomie en tant que dispositif stratégique et méthodologique (Martin et al., 1996) est aussi le lieu d'une construction sociale autant que scientifique. Dans ce cadre, la formation à l'intervention s'inscrit dans une unité entre recherche et pratique. Envisageons les conditions pour que cette proposition soit viable.

37 Une condition parmi d'autres est la reconnaissance que les logiques qui gouvernent la recherche et la pratique sont contradictoires : le critère de vérité structure l'activité scientifique cependant que le critère d'efficacité pilote l'action et la transformation des situations et des organisations. Leur caractère antagoniste gagnerait à être examiné depuis les rythmes de l'action ergonomique. Avançons quelques propositions.

La phase d'analyse et de reformulation de la demande exige une disponibilité et une écoute approfondie afin que s'élabore une première vision systémique de la situation. Elle gagne à ne pas être dépendante d'une activité de problématisation scientifique au risque d'une fragmentation et d'une déconnexion du réel du travail. Pour aller plus loin, cette phase cruciale d'exploration et d'instruction doit en être déconnectée.

La phase de construction d'un diagnostic ancré dans la réalité du travail peut en revanche trouver une mise en écho avec d'autres contributions de praticiens et de chercheurs. L'antagonisme entre recherche et pratique, s'il est flagrant dans la première phase de l'intervention, n'est plus actif ensuite.

40 Si on considère l'intervention dans sa globalité, la production d'outils et de méthodes et le renouvellement de cadres conceptuels est possible du fait de la diffusion dans la communauté. Ils sont ensuite mis à l'épreuve de la pratique, modifiés et progressivement généralisés. Recherche et pratique sont alors dans une dynamique d'enrichissement réciproque. Les propositions que nous formulons ici ne sont pas nouvelles, elles puisent dans des réflexions initiées de longue date dans la discipline, notamment au plan épistémologique (Daniellou, 1996).

41 Ces partis pris explicités, nous allons à présent détailler le dispositif de formation que nous mettons en œuvre. 


\section{Former par la mise en débat des interventions en train de se faire}

42 L'encadrement des interventions des étudiants en entreprise se réalise dans un séminaire bi-mensuel de suivi de stage auquel contribue un collectif d'encadrement composé d'une enseignante chercheure, d'une professeure associée, d'une consultante en ergonomie. Le postulat du séminaire est, nous l'avons énoncé en introduction, que l'outillage précoce des pouvoirs d'agir des apprentis ergonomes est un vecteur du développement de leurs capacités d'agir en ergonomie. Ce postulat se traduit de trois façons :

- Les étudiants sont autonomes sur leurs terrains d'intervention. Corollaire de cette autonomie importante, l'encadrement mis en place à l'université associe des enseignantschercheurs et des consultants ;

- Les encadrants universitaires n'interfèrent pas directement dans la relation que l'étudiant construit avec ses interlocuteurs sur le terrain dès la réception des offres de stages jusqu'à la fin de son intervention et la soutenance publique de son travail ;

- Le guidage des encadrants à l'université s'inscrit dans une perspective de co-activité en faisant une large place au collectif étudiant.

43 Au rythme de deux séances par mois, le séminaire de suivi réunit l'ensemble des étudiants ainsi que les trois encadrants universitaires: un professeur PAST $^{3}$, un enseignant chercheur et un ergonome professionnel pour une durée de trois heures dans une pièce dédiée à la formation de Master 2.

Il met en présence des acteurs porteurs de logiques contradictoires: les logiques formatives portées par les encadrants universitaires et les logiques de connaissance et d'action portées par les tuteurs en entreprise.

L'encadrement se structure dans l'action solidaire de deux collectifs: le collectif d'encadrement qui inclut praticiens et enseignants-chercheurs et le collectif des étudiants qui devient progressivement acteur de l'avancement du travail de chacun. Responsables et garants de la viabilité des interventions menées avec les acteurs de terrain, de la cohérence du travail d'élaboration de la problématique, l'équipe universitaire outille les praticiens pour conduire leurs interventions et y construire leurs gestes professionnels. Acteurs de leurs interventions et soutien de leurs camarades, les étudiants forment un groupe qui constitue un espace où peut s'expérimenter le don et le contre don qui se concrétise en partage d'expériences, d'outils, de connaissances. L'organisation spatiale en $U$ vise à favoriser les échanges et le dialogue entre les participants.

Chaque séance débute par un tour de table où chacun expose l'état d'avancement de son intervention, ses interrogations, ses difficultés et/ou points de blocage tout en spécifiant s'il compte effectuer une présentation. Puis les présentations se succèdent à raison de deux à trois par séance, elles prennent appui sur des vidéo-projections ou bien des schémas réalisés au tableau. Guidées par les encadrants, ces séances sont l'occasion d'échanges au sein du collectif étudiant. Les questions adressées au collectif trouvent des échos dans les expériences singulières du fait de leur similarité ou au contraire de leur spécificité, des éléments de solution en cours de construction sont mis en partage.

Au-delà de ces deux collectifs, le groupe de suivi en entreprise est un troisième espace de construction collective de l'intervention. Regroupant la diversité des acteurs concernés 
par l'intervention, ce groupe de suivi assure plusieurs fonctions. Il donne une visibilité à l'intervention en élargissant le nombre d'interlocuteurs qui seront actifs dans la construction de la mission. Il amène les membres de ce groupe à prendre position au fur et à mesure de l'avancement de la mission, à réaliser des choix et prendre des décisions (pré-diagnostic et pistes de transformation). Ainsi, les tuteurs en entreprise, non présents dans les séances de travail à l'université, assurent un rôle majeur dans la construction collective de l'intervention sur le terrain (accueil et contact permanent avec l'étudiant, contribution à la construction du groupe, aide à l'animation...).

\subsection{Rendre compte d'une expérience pédagogique, précisions méthodologiques} transition dans le cadre d'un départ à la retraite. Le recueil s'est focalisé sur le relevé systématique des difficultés rencontrées par les étudiants à chaque étape de leurs interventions et des ressources mobilisées par les encadrants. Des extraits de dialogues enseignants-étudiants ont été transcrits permettant de caractériser les situations pédagogiques ;

- Des prises de notes des auteures en situation d'encadrement en relevant de façon similaire les obstacles des étudiants à chaque étape et les ressources des encadrants sur 3 années universitaires, et auprès de trois promotions d'étudiants (de 13 à 17 étudiants par promotion);

- Enfin, un entretien collectif mené auprès d'étudiants à l'issue de la première année d'enseignement, complété de 5 entretiens individuels avec des étudiants disponibles pour s'exprimer sur leur vécu de l'encadrement de stage à l'issue de leur soutenance.

Le matériau récolté s'est avéré relativement homogène. Des éléments récurrents et convergents ont pu être extraits et constitués en catégories d'activité, les verbalisations venant illustrer la catégorisation obtenue.

51 Afin de restituer la mise en œuvre de ce dispositif de formation, nous décrivons dans un premier temps les séances de suivi de stage aux différentes étapes de construction de l'intervention (reformulation de la demande, observations ouvertes, pré-diagnostic, observations systématiques, diagnostic, restitution). Puis nous rendrons compte de deux moments charnières que sont la reformulation de la demande et le pré-diagnostic. Les tensions et réalignements analysés dans ces deux moments clés connaissent deux sortes de dénouements, l'extension de la surface de l'intervention et le rôle croissant du collectif que nous détaillons ensuite.

\subsection{Tensions et alignements dans la construction des interventions}

La prise de contact avec les entreprises et la recherche de stage sont les premières étapes du travail des étudiants. Les encadrants universitaires relaient sans les filtrer les offres de 
stage qu'ils reçoivent, les étudiants en récoltent par eux-mêmes, ils élaborent des candidatures spontanées envers les entreprises ou les institutions. Ils sont guidés en séminaire de suivi de stage pour la prise de contact avec les entreprises, l'analyse de la demande (simulation d'entretien lors de la première rencontre).

Traversé de tensions aux moments clés de l'intervention, ce dispositif de formation évolue au fur et à mesure de la progression de chaque intervention. Le tableau 1 présente la chronologie des étapes et rend compte de l'évolution des tensions et des relâchements entre les trois grands types d'acteurs que sont les tuteurs en entreprise, les étudiants et les encadrants universitaires.

Tableau 1 : Tensions et alignements dans le dispositif de formation. Table 1: Tensions and alignments in the training device

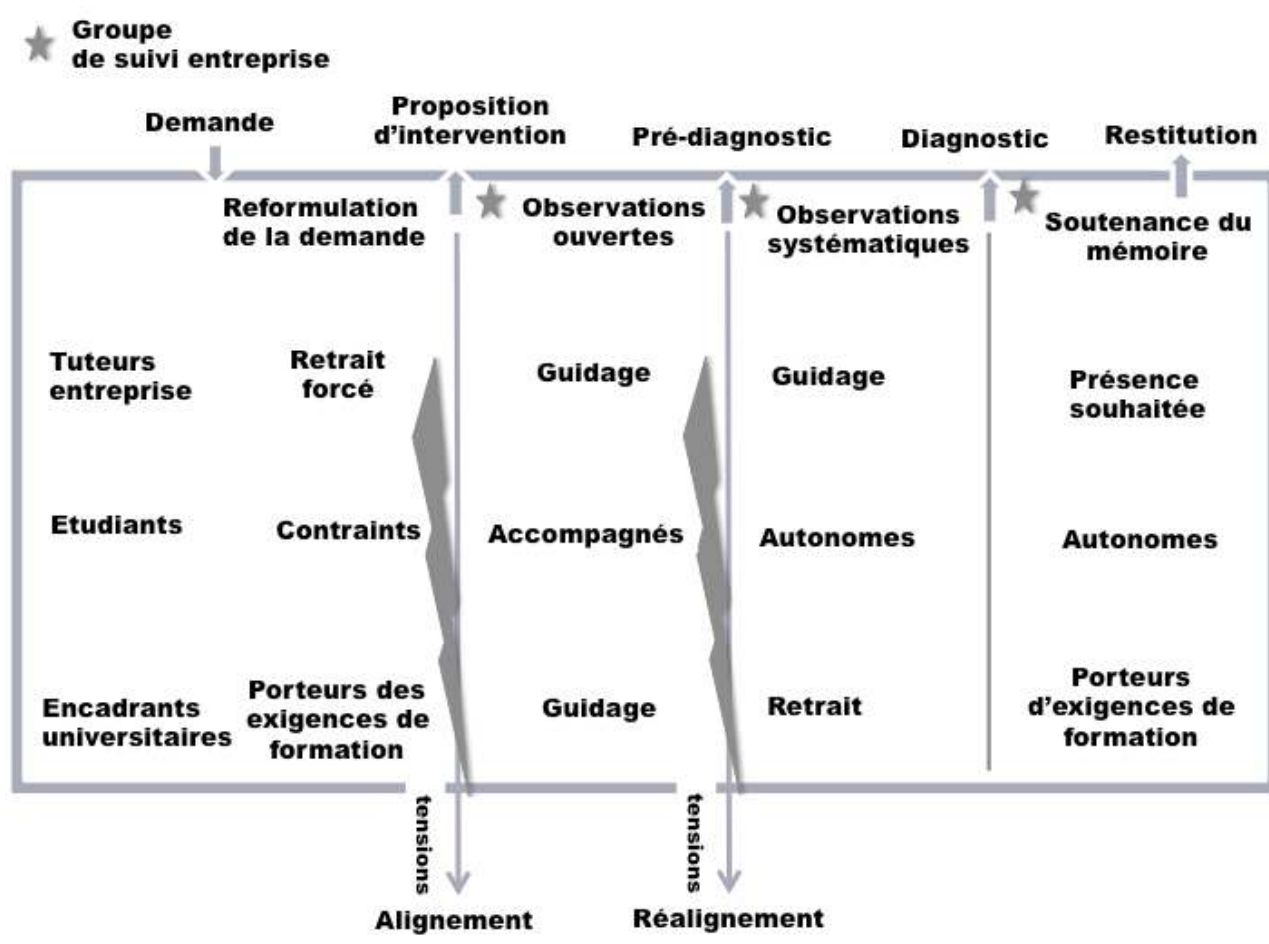

Tout au long des interventions, les postures des acteurs, tuteurs et encadrants universitaires, oscillent entre accompagnement et retrait.

Les étudiants sont fortement accompagnés par le dispositif pédagogique dans les premières phases de leurs interventions et sont plus autonomes ensuite. La réception des offres et leur instruction, la définition des objectifs et du périmètre de la mission ainsi que la constitution du groupe de suivi sont des moments clés. Un guidage et la mobilisation d'un outillage adéquat sont nécessaires.

Au fur et à mesure de l'avancement, le collectif étudiant prend une place de plus en plus importante et les constructions avec les acteurs de terrain étayent, interrogent et enrichissent la mission. Ainsi un ensemble de ressources diversifié se construit dans les différents espaces collectifs de l'intervention. Il conduit à un retrait progressif des encadrants qui va de pair avec une plus grande autonomie des étudiants.

Les tuteurs en entreprise sont présents tout le long de l'intervention. Ils sont maintenus à distance par le dispositif pédagogique dans la première phase d'instruction et de reformulation de la demande, ils sont acteurs du collectif qu'est le groupe de suivi qui 
entre en écho avec le collectif encadrants-étudiants et le collectif étudiant. Ils évoluent d'un retrait forcé vers une posture de guidage de l'étudiant et de prise de parole souhaitée lors de la soutenance publique des travaux.

Lieu où les étudiants arrivent chargés, riches, ou tendus de leurs expériences accumulées au sein de l'entreprise, le suivi de stage permet l'expression collective de ces tensions et nous allons examiner les deux moments charnières évoqués précédemment, la reformulation de la demande et la construction du prédiagnostic.

\subsection{Les tensions intrinsèques à la reformulation de la demande}

La reformulation de la demande est une phase complexe pour les jeunes ergonomes car il s'agit de mettre en œuvre une reformulation en un problème traitable en ergonomie, et ce faisant, de construire un lien avec le tuteur accueillant le stage, de rendre visible l'existence d'une intervention en ergonomie, et de gagner la confiance des interlocuteurs auprès desquels une diversité d'informations doivent être recueillies.

Une partie des difficultés rencontrées tient aux logiques divergentes portées par les acteurs, elles suscitent une mise en tension au cœur de laquelle se trouvent les étudiants :

- Les tuteurs en entreprise souhaitent recruter un bon étudiant parmi plusieurs candidats, capable de répondre à leur demande et de remplir une mission précise ;

- Les étudiants sont dans la crainte de ne pas trouver de stage. L'apparition d'une offre de stage suscite l'envie de commencer le stage le plus tôt possible et de passer outre le travail d'instruction et de reformulation;

- Les encadrants sont attentifs à l'exploration de l'offre de stage par les étudiants et sa constitution en une demande reformulée ouvrant vers une mission viable.

61 Ces logiques divergentes peuvent être caractérisées en termes de discordance des objets d'activité à partir des propositions de Vygostky puis Leontiev (Kaptelinin, 2005). Sans entrer dans le détail des constructions conceptuelles, nous retiendrons que l'objet de l'activité est autant ce vers quoi l'activité est orientée (predmet) que le support externe de celle-ci (objekt). L'ambiguïté constitutive de la notion d'objet d'activité exprime le fait qu'il n'est pas une chose uniquement extérieure au sujet, il le met directement en rapport avec lui-même exprimant ainsi la relation dialectique qui unit l'homme au monde: mobile qui incite à agir autant que but vers lequel l'activité est dirigée. L'examen historique auquel se livre Kaptelinnin le conduit a qualifier l'objet d'activité « as a sense maker which gives meaning to and determines values of various entities and phenomena » (Kaptelinin, op. cit. p. 5).

Saisis dans le cadre de ce texte en tant que "predmet", les objets d'activité des acteurs parties prenantes du suivi de stage sont divergents à cette étape de reformulation de la demande. Cette divergence est l'occasion de tensions qui évoluent et se relâchent à l'occasion d'une intégration progressive. Le tableau 2 en présente la dynamique, de la divergence vers l'intégration. 
Tableau 2 : De la demande à sa reformulation : acteurs et objets d'activité.

Table 2: From demand to reformulation : actors and objects of activity

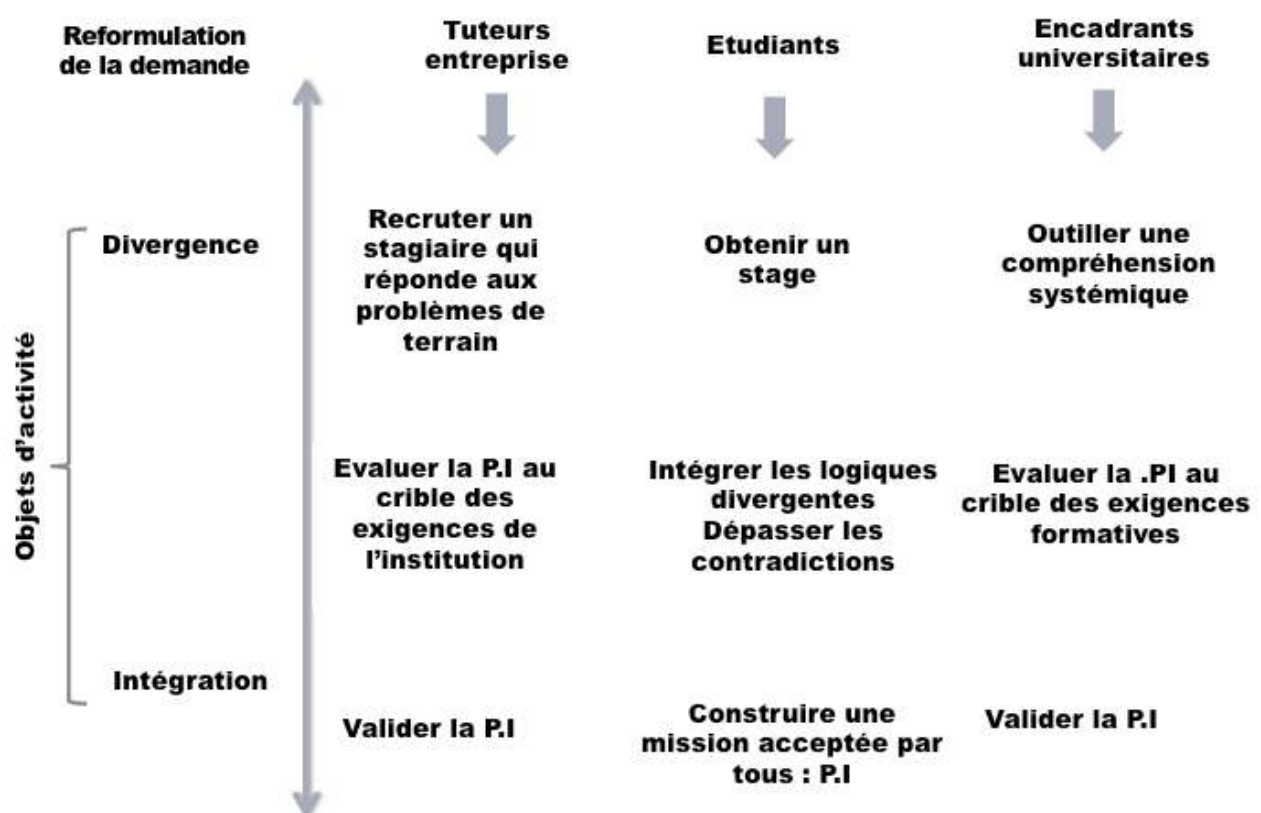

63 La divergence des objets d'activité provoque une mise en tension au cœur de laquelle se trouvent les étudiants. Une double mission s'ouvre à eux. Il s'agit de construire et de conduire l'intervention par une compréhension des acteurs, de leurs enjeux et des problèmes qu'ils s'expriment (analyse sociale) et une appréhension des activités de travail concernées, leurs déterminants leurs effets et les solutions déjà mises en place (analyse causale).

Or à ce stade initial, les étudiants ne sont pas convaincus de l'importance d'instruire les offres de stage ainsi que l'exprime une étudiante récemment diplômée : « Nous sommes stressés, tout ce que nous voulons, c'est un stage. Alors l'analyse sociale et causale, c'est un exercice, je l'ai mené de manière scolaire, je ne comprenais pas pourquoi vous trouviez cela si important. À ce moment-là, on vous en veut de nous empêcher de rentrer directement en stage ».

En outre, en tant que praticiens débutant en ergonomie il se sentent difficilement porteurs des concepts, des méthodes, des principes et des valeurs de la discipline ainsi que le verbalise une étudiante lors d'une séance de suivi de stage : «Le temps avance et c'est pas que je vois pas la fin, c'est que je ne vois pas le début!», exprimant ses incertitudes quant à sa capacité à mener une mission en ergonomie.

Le dénouement des tensions passe par l'élaboration par l'étudiant d'une proposition d'intervention permettant de créer de la cohérence et une intégration entre les objets de l'activité des acteurs. Ainsi, l'étudiant travaille à résoudre une situation tendue dont il est le protagoniste, la reformulation de la demande s'accomplit dans la construction d'une convergence des objets de l'activité de ses divers interlocuteurs. Au travers de l'établissement d'une proposition d'intervention (P.I.) partagée et validée par les trois parties, l'étudiant obtient ce vers quoi il tend depuis le départ, à savoir l'entrée en stage. 
67 À l'issue de la reformulation de la demande, les étudiants s'engagent dans leurs observations ouvertes. La tension se relâche, ils œuvrent en se confrontant à la complexité du réel. Cependant, ce n'est que temporaire puisque quelques semaines après se pose la question du pré-diagnostic qui va de pair avec la ré-émergence des tensions.

\subsection{Les tensions intrinsèques au pré-diagnostic} ressources. L'étudiant a une bonne connaissance de l'activité, en revanche, il a peu d'expérience de l'intervention ergonomique. Les enseignants ont une diversité d'expériences d'interventions ergonomiques (recherche-action, études et expertises CHSCT, conduite de projet). Le groupe de suivi est ancré dans l'organisation, en a une expérience en termes de fonctionnement, il peut aussi avoir une pratique du débat collectif. Il permet à l'étudiant de distinguer les éléments d'analyse qui font consensus au sein de l'entreprise de ceux qui constituent des points durs voire des chantiers de réflexion et d'action. Quant aux encadrants, ils aiguillent l'étudiant dans une compréhension systémique du terrain grâce à leur connaissance d'une diversité d'interventions.

70 À cette étape la compréhension du travail, nourrie des rencontres avec les acteurs et des observations ouvertes, a permis d'identifier les sources de variabilité et de diversité gérées par les opérateurs et d'identifier les problèmes rencontrés. Le pré-diagnostic est une problématisation de cette compréhension sous un jour nouveau. Il produit un ensemble d'hypothèses, définit les méthodologies et les observables retenus en vue des observations systématiques et envisage les pistes de transformation possibles. La difficulté réside dans le changement de niveau qui est exigé comme le suggèrent Vézina et Baril (2009) : il s'agit de passer d'un registre descriptif à un registre plus explicatif qui cible les situations de travail concernées et les déterminants à retenir et oriente les pistes de transformations futures probables.

71 Arrive enfin l'étape de soutenance des travaux après un dépôt auprès des encadrants universitaires. Les enseignants (re)découvrent l'intervention ergonomique dans son ensemble, les tuteurs en entreprise sont invités aux soutenances dans lesquelles leur parole est attendue. Lorsque l'intervention ergonomique remplit les exigences de formation et les attentes des tuteurs alors l'étudiant est diplômé en ergonomie.

Ainsi les tensions qui apparaissent et se résolvent tout au long de l'année révèlent les contradictions des différents acteurs du système au cœur desquelles se joue, à chaque fois pour l'étudiant le devenir de son intervention. En dépassant les contradictions incarnées dans la disparité des objets de l'activité des acteurs, l'étudiant acquiert un point de vue systémique, et dénoue les tensions à l'œuvre provoquant un relâchement du système dans son ensemble. Une fois que le prédiagnostic, méthodes et observables constituent des propositions qui conviennent à l'ensemble des acteurs, alors les observations systématiques peuvent se dérouler, les rails de l'intervention ergonomique sont désormais en place. 


\subsection{Extension de la surface de l'intervention et rôle croissant du collectif}

73 L'apaisement des tensions que nous venons de décrire ouvre deux voies, celle d'une extension de la surface de l'intervention à travers le rôle actif du groupe de suivi en entreprise et le collectif étudiant. Au sein du collectif étudiant, chacun/e devient peu à peu capable d'être partie prenante des autres interventions et d'en débattre collectivement.

74 Espace de mise en partage et de mise en visibilité de l'intervention, le groupe de suivi contribue à élargir la surface d'intervention de plusieurs façons. Il relie l'étudiant à son terrain via un réseau d'acteurs élargi. Ce faisant il étend le binôme initial tuteurergonome en stage permettant au jeune praticien de gagner en indépendance. Il est le lieu de la mise au travail de l'intervention en train de se faire. L'intervention « en tant que matière première » est l'occasion d'engager un débat sur le travail, "de dire et d'entendre dire » ce qui fait désaccord et de construire une vision partagée de ce qui est prioritaire.

75 Un autre ingrédient est propice à l'élargissement de la surface d'intervention, il s'agit de la constitution du collectif étudiant. Le temps contraint (une séance de 3 heures bimensuelle) peut produire une certaine frustration des étudiants de ne pas avoir toujours le temps de raconter leurs terrains dans le détail ${ }^{4}$. Leur engagement dans leurs interventions les pousse à constituer un collectif d'entraide dans lequel les plus avancés aident leurs collègues qui ont trouvé un stage plus tardivement.

76 Les enseignants encouragent l'existence de ce collectif de travail. Lors des séances de suivi, ils sont invités à tisser des liens entre leurs terrains et la nature des problèmes rencontrés par les autres étudiants, à travers des échanges au-delà du suivi de stage en raison de la proximité des problématiques ergonomiques rencontrées.

Enfin, les conditions matérielles sont favorables à la constitution d'un collectif étudiant. La promotion dispose d'une salle dédiée tout au long de l'année. Acteurs dans l'organisation logistique du master (matériel, clef de la salle), les étudiants bénéficient de casiers et sont invités à s'approprier les lieux à leur guise, en affichant des documents sur les murs. Au fur et à mesure de l'année, ils prolongent le travail commun après les séances de suivi de stage.

78 Notons que si les conditions restent inchangées, le collectif étudiant n'est pas toujours aussi vivace d'une année sur l'autre.

79 Tout au long de l'année, l'intervention est rythmée par des mouvements d'expansion et de resserrement. Dans la rencontre entre ce qui se tient face à lui, existe et lui résiste le praticien en formation construit son geste professionnel au sein de trois espaces de construction collective qui s'étayent progressivement : le collectif encadrants-étudiants à l'université qui prend place dans les premiers temps, puis le groupe de suivi qui se construit dès l'entrée en stage, et enfin, le collectif des étudiants qui prend forme au fil des mois.

80 Plaçons-nous à présent au plan de l'interaction entre les encadrants et les étudiants pour explorer la co-activité dans laquelle ces acteurs s'engagent. 


\section{Co-activité encadrants-étudiants}

81 La co-activité encadrants-étudiants s'inscrit dans une asymétrie de départ entre enseignants et étudiants qui va évoluer au fil du temps et se résorber. Elle connait différentes phases, de fluidité ou au contraire d'enlisement.

\subsection{Asymétrie entre les acteurs}

82 L'asymétrie entre étudiants et encadrants, caractéristique de toute situation formative, se décline ainsi. Les étudiants ont accès au terrain, aux acteurs, ils en éprouvent la réalité. En revanche, ils ont une vision fragmentée des problèmes exprimés, ils sont invités par les enseignants à étayer leur recueil d'information et tout élément utile à leur compréhension. Ils sont contraints, voire empêchés par les enseignants de s'engager et de contractualiser une mission avec leurs interlocuteurs tant qu'ils échouent à construire une vision globale et systémique et à saisir le bon niveau de granularité de l'activité.

Les encadrants, bénéficient d'une grille de lecture nourrie de leurs expériences d'interventions en ergonomie. Cette diversité d'expériences associées à une expérience d'enseignement les dote d'une certaine agilité pour déceler dans les interventions en train de se construire les lacunes, les écueils ou les impasses dans lesquelles peuvent se trouver les étudiants. Toutefois, n'ayant pas accès aux terrains, ils ne peuvent en construire une compréhension immédiate, elle est au contraire médiatisée par les étudiants.

La co-activité encadrants-étudiants s'apparente donc à une situation de double aveugle, sans prise avec le terrain pour les enseignants, et avec une faible maitrise de la démarche et de la discipline pour les étudiants. Pour que ce dispositif pédagogique soit fructueux, il est nécessaire que :

- Les étudiants donnent suffisamment à voir, entendre et sentir leurs terrains aux encadrants et aux autres étudiants ;

- Les encadrants développent une écoute fine de «ce qui se dit» et de «ce qui est tu» et proposent un guidage permettant aux étudiants de cheminer à leur rythme dans une compréhension de leur situation de terrain et une appropriation de la démarche ergonomique.

De ce maillage fin des capacités de chacun à mobiliser ses ressources expérientielles, conceptuelles et d'écoute, à les mettre en partage et en débat découle la possibilité pour les étudiants de s'emparer progressivement de leur intervention et d'étayer celles de leurs camarades.

Ces oscillations s'accomplissent dans la spécificité de chaque terrain d'intervention où l'exercice autonome du pouvoir d'agir de chaque apprenti ergonome vient mettre à l'épreuve, interroger et étayer la construction de ses capacités. Les ressources construites par les jeunes praticiens puisent dans la diversité de leurs expériences, s'élaborent en des actes qui délient et relient différemment histoires et vécu, elles se nourrissent de réflexivité dans une construction lente, hésitante et jamais linéaire.

87 Examinons à présent la dynamique de cette co-activité. 


\subsection{Dynamique de la co-activité, entre fluidité et enlisement} rythmes des apprentissages seront divers et variables, et se préparer à la survenue de points de rupture, temporaires et très rarement définitifs. Cet espace de développement est un espace du devenir capable, les formes qu'il peut prendre chaque année ne sont pas totalement prédictibles. L'action des jeunes praticiens, réglée par l'outillage proposé, n'en est que partiellement déterminée.

Afin de mieux saisir cette co-activité, notons que les oscillations des étudiants que les encadrants guident suivent un mouvement qui va d'une adhérence à la demande initiale vers une posture de distanciation; d'une vision figée et locale vers une perspective systémique de l'intervention; d'un point de vue fragmenté de l'activité et du travail vers un point de vue global, continu et multidimensionnel.

Pour donner à voir la dynamique de la co-activité, nous choisissons deux types de situations, celles où la co-activité est fluide et celles où des blocages apparaissent, caractéristiques d'un enlisement. Il sera transitoire, évoluant vers des formes de coactivité au fur et à mesure des séances et des échanges collectifs.

\section{Déroulement d'une séance}

91 La présentation de l'étudiant au collectif de suivi de stage, d'une durée de 15 à 20 minutes, peut porter sur l'analyse de la demande, les observations ouvertes, ou le pré-diagnostic hypothétique. Au cours de ces présentations formelles, les étudiants donnent à voir l'avancement de leur travail, les résultats d'analyses sociales et/ou causales, les résultats d'observations (tableaux, chroniques d'activité...) ainsi que des documents de terrain (photographies, plans).

92 Pendant la présentation par l'étudiant, les encadrants cherchent à s'immerger suffisamment dans la situation pour pouvoir guider l'étudiant. Les autres étudiants cherchent également à comprendre la situation présentée, la comparent avec les situations de leurs collègues et celles auxquelles ils sont eux-mêmes confrontés.

Le tableau n ${ }^{\circ} 3$ présente les activités des encadrants et des étudiants structurées en trois temps : la présentation (Temps 1), les échanges et les débats (Temps 2), la co-construction (Temps 3). 
Tableau 3 : co-activité des enseignants et étudiants.

Table 3 : Teacher and student coactivity

\begin{tabular}{|c|c|c|c|}
\hline $\begin{array}{l}\text { Séance de } \\
\text { suivi de stage }\end{array}$ & $\begin{array}{l}\text { Activité étudiant } \\
\text { qui expose }\end{array}$ & Activité encadrants & $\begin{array}{l}\text { Activités étudiants } \\
\text { auditeurs }\end{array}$ \\
\hline $\mathrm{T} 1$ & $\begin{array}{l}\text { Rend compte de l'avancement de } \\
\text { son intervention et explicite les } \\
\text { caractéristiques de son terrain } \\
\text { (acteurs, problèmes, contexte, } \\
\text { organisation, santé...) } \\
\begin{array}{l}\text { Ressources: photos, plans, } \\
\text { schémas, tableaux, outils } \\
\text { d'objectivation) }\end{array}\end{array}$ & $\begin{array}{l}\text { Immersion en double écoute : } \\
\text { - Appréhender le terrain de l'étudiant } \\
\text { Ressources : questions de compréhension } \\
\text { - Comprendre la progression de l'étudiant } \\
\text { Ressource : écoute des silences, des écarts, } \\
\text { des contradictions, }\end{array}$ & $\begin{array}{l}\text { Écoute active : } \\
\text { s'immerger, comprendre le } \\
\text { terrain de l'étudiant en } \\
\text { comparant et en le } \\
\text { confrontant à ses problèmes }\end{array}$ \\
\hline $\mathrm{T} 2$ & Répond aux questions & $\begin{array}{l}\text { Guidage } \\
\text { Ressources : relances interrogatives, } \\
\text { questions, réouverture de possibles }\end{array}$ & $\begin{array}{l}\text { Echanges } \\
\text { Ressources : questions de } \\
\text { compréhension, } \\
\text { comparaison avec d'autres } \\
\text { situations }\end{array}$ \\
\hline T3 & \multicolumn{3}{|c|}{$\begin{array}{l}\text { Reformuler et ancrer l'intervention dans le travail et l'activité } \\
\text { Adopter un point de vue systémique et dynamique de l'intervention en train de se faire }\end{array}$} \\
\hline
\end{tabular}
fréquentes: questions de compréhension, identification de thématiques d'échanges pouvant donner lieu à une mise au point plus générale (éthique ou déontologie, mise en relief du caractère plus général du cas). Puis un temps de co-construction s'engage (T3) dans lequel l'étudiant, les encadrants et le collectif étudiant se déplacent dans l'espace l'intervention pour explorer les ouvertures possibles ou les contraintes ou impossibilités. Ils effectuent des simulations pour statuer ensemble sur la meilleure manière de positionner l'intervention.

作 étudiants qui exposent ainsi que par l'alternance des prises de parole. Lorsque la coactivité est fluide, le collectif peut déboucher sur une co-activité forte, séquence fructueuse. En revanche lorsque malgré plusieurs itérations entre la présentation (T1) et les échanges et débats (T2), la co-activité ne débouche pas sur une co-construction (T3), alors les enseignants alertent sur le risque d'impasse encouru, invitent à une exploration plus approfondie avant d'engager la phase ultérieure de l'intervention, donnant parfois lieu à des insatisfactions de la part de l'étudiant concerné.

Examinons les deux types de situation.

\section{La fluidité de la co-activité}

Dans cette configuration, l'étudiant partage son expérience du terrain avec le collectif, il en restitue sa compréhension au moment T1. Les enseignants alternent une posture d'incitation des étudiants à s'exprimer avec une posture de délivrance d'éléments conceptuels et méthodologiques. Ils relient l'intervention en cours de présentation et d'autres interventions d'étudiants. Les étudiants auditeurs s'imprègnent du terrain, posent des questions et comparent l'intervention présentée avec leur propre intervention. 

maintenant. l'activité.

Lorsque la co-activité enseignants-étudiants est fluide, elle débouche sur une co-activité forte où l'intervention ergonomique est en expansion. Pétrie par les questions des uns et des autres, elle s'accroit et des pans supplémentaires se dessinent. Les encadrants viennent nourrir, soutenir, étayer l'expérience.

Dans cette configuration, on observe le rôle amplificateur du collectif étudiant qui prend différentes formes. Il peut être porteur de questions, il s'approprie et reformule ce qui est dit par l'encadrant. Ou bien, observateur de la situation d'interaction entre l'étudiant qui expose et les encadrants sans y prendre part. Ou encore, partie prenante des échanges en se projetant dans la posture de l'étudiant qui a la parole ou dans la posture de l'encadrant qui cherche à s'immerger dans la situation et à aiguiller l'étudiant qui a la parole.

Cependant cette configuration de co-activité fluide n'advient pas d'emblée, elle se construit pas à pas et, pendant une période plus ou moins longue, les étudiants tâtonnent pour appréhender le bon niveau de granularité de l'activité, construire un point de vue systémique et ancrer une dynamique d'intervention dans le travail. Ces moments-là se manifestent en suivi de stage par une co-activité qui s'enlise que nous allons explorer

\section{Co-activité et enlisement}

Les moments d'enlisement sont des moments pédagogiques très riches qui révèlent les ingrédients indispensables pour que l'intervention ergonomique puisse progresser à l'étape suivante. Décrivons plus précisément ce qui se passe dans ces situations où une double écoute et un double diagnostic structurent l'activité des enseignants.

Tableau 4 : Co-activité et enlisement. Table 4: Coactivity and sticking points

\begin{tabular}{|c|l|}
\hline Objets de l'activité & \multicolumn{1}{c|}{ Activité des encadrants } \\
\hline Double écoute & $\begin{array}{l}\text { - S'immerger dans le terrain de l'intervention } \\
\text { - Repérer les dissonances, les incohérences, les hésitations } \\
\text { - Ecouter les silences }\end{array}$ \\
\hline Double diagnostic & $\begin{array}{l}\text { - Construire et porter un point de vue systémique et dynamique ancré dans } \\
\text { la situation d'intervention } \\
\text { - Repérer la posture de l'étudiant }\end{array}$ \\
\hline
\end{tabular}

02 La double écoute vise à s'imprégner du terrain, le double diagnostic se saisit des matériaux rapportés, le plus souvent épars et fragmentés, pour y construire une intelligibilité plus globale, expliciter le caractère systémique qui relie ce qui est présenté de façon disjointe et mettre en relief la dynamique de l'intervention ancrée dans

Les encadrants repèrent des dissonances, décèlent les écarts entre ce que dit l'étudiant et ce qu'il affiche, projette ou formalise, ce qu'il tait et les hésitations qui sont les siennes. À cette occasion sont vérifiées la posture de l'étudiant auprès des acteurs, la référence qu'il 
construit au travail réel et à l'activité et la cohérence du positionnement progressif de l'intervention.

Précisons les difficultés les plus fréquemment identifiées par les encadrants au cours de leur activité de double écoute et de double diagnostic :

- Une instruction faible de la demande réalisée de manière mécanique et/ou scolaire ;

- Une élimination des acteurs considérés sans rapport avec la problématique construite ;

- Un ancrage dans l'activité fluctuant et/ou non stabilisé et l'adoption du vocabulaire et des perspectives du demandeur;

- Un discours de compréhension générale du travail rattaché hâtivement à un ensemble de travaux scientifiques ;

- Un discours trop lisse, gommant les paradoxes et les contradictions.

En situation de double écoute, les enseignants manquent parfois de matière pour appréhender globalement la situation et en saisir la dynamique. Dans ce cas-là, de nombreuses questions de compréhension cherchent à éclairer les différents plans de l'intervention et y construire une continuité.

Notons ici que ces moments d'enlisement connaissent des évolutions au fil des séances, du blocage vers une réouverture permettant à une co-activité de se déployer à nouveau.

Le guidage auquel les enseignants se livrent se structure à partir de ce qu'ils perçoivent des écarts et des dissonances dans les présentations des étudiants et les échanges qui ont lieu. Envisageons en détail les registres et les ressources du guidage.

\subsection{Registres et ressources du guidage}

108 Le guidage proposé s'inscrit dans une démarche pédagogique qui s'avère proche de l'accompagnement maïeutique initié par les travaux de Desroches récemment ré-éclairés par Lago (2009). La formation est accompagnement et initiation, elle s'inscrit dans une expérience dense et intensive d'apprentissage dans laquelle un espace de disponibilité et d'exploration, de mise en tension des savoirs et des techniques de métier, de débats et d'appropriations progressives, est proposé. Les encadrants universitaires établissent des passerelles entre les éléments reportés pour permettre à chaque praticien de construire et de conduire son intervention de façon autonome. Ce guidage comprend plusieurs registres d'accompagnement et un ensemble de ressources que nous allons maintenant examiner. Des extraits de verbalisations viendront illustrer notre propos.

\section{Déplacer l'étudiant de son point de vue}

Ce guidage consiste à déplacer de manière indirecte le point de vue de l'étudiant à travers l'utilisation de métaphores, mais également en accentuant son point de vue au travers de caricatures, ou encore en projetant l'étudiant dans un jeu de rôle ou dans un temps ultérieur de l'intervention ergonomique dans l'usage de prolepse ${ }^{5}$. 
Tableau 5 : Accompagner le déplacement de point de vue.

Table 5: Accompanying a shift in point of view

\begin{tabular}{|c|c|c|}
\hline $\begin{array}{c}\text { Registre du } \\
\text { guidage }\end{array}$ & Type de ressources & Extraits de verbalisations \\
\hline \multirow{4}{*}{$\begin{array}{l}\text { Déplacer } \\
\text { indirectement } \\
\text { l'étudiant de } \\
\text { son point de } \\
\text { vue }\end{array}$} & Métaphore & $\begin{array}{l}\text { "L'intervention c'est comme le mouvement d'une méduse, une fois } \\
\text { que le contrat est signé, ouverture pour les observations ouvertes, } \\
\text { resserrement pour le pré-diagnostic " } \\
\text { "Le prédiagnostic doit fonctionner comme un aimant en polarisant } \\
\text { tous les fragments des observations ouvertes » }\end{array}$ \\
\hline & Caricature & "Vous ne serez jamais préventeur, soyez ergonome » \\
\hline & Jeu de rôle & « Si je suis décideur, je dis on change les EPI » \\
\hline & Prolepse & $\begin{array}{l}\text { "Le diagnostic et le prédiagnostic font partie de ce qu'on laisse dans } \\
\text { l'entreprise. À l'arrivée, on se met d'accord, et on tisse dans le } \\
\text { mouvement de l'entreprise. Quand on s'en va c'est un mouvement de } \\
\text { déliaison, il faut penser à ce qu'on laisse derrière nous, à la manière } \\
\text { dont les acteurs vont pouvoir s'emparer du travail de l'ergonome" }\end{array}$ \\
\hline
\end{tabular}

110 Métaphore et proplepse permettent de caractériser l'intervention dans sa globalité, sur le plan de sa construction, pour en donner à voir la dynamique interne, et, sur le plan de sa conduite pour y expliciter la mise en mouvement des acteurs. Caricature, jeu de rôle forcent le trait et invitent à interroger l'action et la posture qui la sous-tend.

\section{Accompagner une réouverture des possibles}

111 Il s'agit dans ce guidage d'accompagner l'étudiant en l'alertant, l'invitant à explorer autrement les contraintes et à ré-ouvrir les possibles à partir de ce qui semble clos et fermé.

Tableau 6 : Accompagner une réouverture des possibles.

Table 6: Accompanying a reopening of possibles

\begin{tabular}{|c|c|c|}
\hline $\begin{array}{l}\text { Registre du } \\
\text { guidage }\end{array}$ & $\begin{array}{l}\text { Type de } \\
\text { ressources }\end{array}$ & Extraits de verbalisations \\
\hline \multirow{3}{*}{$\begin{array}{l}\text { Accompagner } \\
\text { une réouverture } \\
\text { des possibles }\end{array}$} & Alerter & $\begin{array}{l}\text { "L'endroit où il ne faut pas aller, c'est quand on oublie l'activité " } \\
\text { "Attention à la manière dont vous présentez le responsable comme si les } \\
\text { difficultés étaient liées aux caractéristiques de la personne. " } \\
\text { Qui dit cela? Vous ou vos interlocuteurs?... Ah ! vous n'êtes pas maitre à } \\
\text { bord?" } \\
\text { "Votre parole ressemble à la parole d'un chef qui dit ce qu'il faudrait } \\
\text { faire" }\end{array}$ \\
\hline & $\begin{array}{l}\text { Explorer les } \\
\text { contraintes }\end{array}$ & $\begin{array}{l}\text { «Il faut tenter de changer de position même lorsque vos interlocuteurs } \\
\text { attendent que l'ergonome soit un exécutant» } \\
\text { "Ne vous enfermez pas dans les pistes de solution » }\end{array}$ \\
\hline & $\begin{array}{l}\text { Réouvrir les } \\
\text { possibles }\end{array}$ & $\begin{array}{l}\text { "On ne généralise pas en épuisant le réel mais en montrant son caractère } \\
\text { représentatif par rapport au problème posé » } \\
\text { «L'analyse des enjeux c'est important pour voir jusqu'où ça peut mener, } \\
\text { ça pointe les leviers » } \\
\text { "Posez des questions naïves aux opérateurs : racontez-moi comment vous } \\
\text { travaillez » }\end{array}$ \\
\hline
\end{tabular}

112 Des alertes peuvent être formulées en cas d'oubli du caractère central de l'activité ou d'éviction du couplage homme-situation au profit d'une caractérisation des personnes ou 
encore de difficulté à être dans la posture du praticien ergonome. L'exploration des contraintes et l'ouverture des possibles concernent autant les modalités pratiques, dans ses contacts avec les opérateurs et les utilisateurs, que les dimensions plus méthodologiques ou stratégiques de l'intervention.

\section{Consolider l'ancrage dans le travail réel et l'activité}

Veiller à l'ancrage continu dans le travail réel et l'activité consiste à désagglomérer ce qui est confus chez l'étudiant ou au contraire réorienter et enrichir ce qui est insuffisamment creusé.

Tableau 7 : Consolider l'ancrage dans le travail réel et l'activité. Table 7: Strengthening anchoring in work and activity

\begin{tabular}{|l|l|l|}
\hline $\begin{array}{c}\text { Registre de } \\
\text { guidage }\end{array}$ & $\begin{array}{c}\text { Type de } \\
\text { ressources }\end{array}$ & \multicolumn{1}{c|}{ Extraits de verbalisations } \\
\hline $\begin{array}{l}\text { Consolider } \\
\text { l'ancrage dans } \\
\text { l'activité }\end{array}$ & Pointer & $\begin{array}{l}\text { «Il existe plusieurs interventions possibles et on fait des choix " } \\
\text { "Les propos idéologiques tels que "les opérateurs sont de mauvaise foi" } \\
\text { doivent être mis de côté " } \\
\text { "C'est bien vous avez une bonne vue d'ensemble mais il faut redescendre } \\
\text { pour atteindre le bon niveau de granularité de l'activité " } \\
\text { "Mais que font les opérateurs? vos descriptions sont trop fragmentées, } \\
\text { elles ne donnent pas à voir l'activité " }\end{array}$ \\
\hline
\end{tabular}

114 Le pointage permet de repérer les lacunes identifiées par un recentrage sur le travail et l'activité. Il concerne autant le périmètre de la mission que l'appréhension de la réalité du travail et la nécessité de renoncer en faisant un choix. C'est au travers de cet ancrage dans l'activité que se joue le positionnement de l'ergonome.

\section{Stopper la progression en cas d'impasse}

En cas d'enlisement, l'avancement du travail peut être stoppé à des fins d'exploration plus approfondie. C'est le cas lorsque le caractère fragmentaire de la vision que porte l'étudiant dans son intervention perdure.

Tableau 8 : Stopper la progression en cas d'impasse.

Table 8: Stopping progress in cases of stalemate

\begin{tabular}{|c|l|l|}
\hline $\begin{array}{c}\text { Registre de } \\
\text { guidage }\end{array}$ & $\begin{array}{c}\text { type de } \\
\text { ressources }\end{array}$ & \multicolumn{1}{|c|}{ Extraits de verbalisations } \\
\hline $\begin{array}{c}\text { Stopper la } \\
\text { progression en } \\
\text { cas d'impasse }\end{array}$ & $\begin{array}{l}\text { Ré- } \\
\text { orientation } \\
\text { redirection }\end{array}$ & $\begin{array}{l}\text { «Votre prédiagnostic est fragmenté, non systémique il ne tient rien } \\
\text { ensemble pour le moment, je vous propose d'arrêter et de le retravailler. } \\
\text { Vous le représenterez la prochaine fois " }\end{array}$ \\
\hline
\end{tabular}

Dans ces cas, les étudiants sont invités à reprendre leurs notes, leurs écrits et leurs premières observations afin de les mettre en écho et d'y construire les pistes d'une première continuité.

\section{Rassurer, donner confiance}

Les différents guidages que nous venons d'évoquer rendent compte d'un engagement réciproque des étudiants et des enseignants. Fortement articulé à l'ensemble des autres 
registres, le guidage par réassurance est primordial, il réitère le contrat de confiance et l'engagement réciproque des étudiants et des enseignants. Il permet également aux autres guidages d'être effectifs.

Tableau 9 : Rassurer, donner confiance.

Table 9: Giving confidence and assurance

\begin{tabular}{|l|l|l|}
\hline \multicolumn{1}{|c|}{$\begin{array}{c}\text { Registre de } \\
\text { guidage }\end{array}$} & $\begin{array}{c}\text { type de } \\
\text { ressources }\end{array}$ & \multicolumn{1}{c|}{ Extraits de verbalisations } \\
\hline $\begin{array}{l}\text { Rassurer, donner } \\
\text { confiance }\end{array}$ & Conseil & $\begin{array}{l}\text { «Il ne faut pas avoir peur des questions naïves, c'est indispensable pour } \\
\text { comprendre le processus technique auquel vous avez affaire là » } \\
\text { « Ce n'est pas vous qui êtes en cause, on discute travail et positionnement } \\
\text { de l'intervention » }\end{array}$ \\
\hline
\end{tabular}

118 Il s'agit donc autant d'encourager l'action que d'expliciter la différence entre soi et son travail. Ce guidage est mobilisé systématiquement et vient en appui des autres modes de guidage qui sont parfois subjectivement exigeants.

119 L'issue des échanges lors du suivi de stage n'est pas toujours le même, entre prise de conscience quasi immédiate ou à distance des séances, elle voit des co-constructions collectives se mettre en place à des rythmes différenciés, permettant à chacun de progresser à son rythme dans son intervention.

Les quelques situations que nous avons rapportées donnent à voir les modalités de guidage dans une action indirecte sur la relation de l'étudiant à son intervention, en l'incitant à se rapprocher, à reculer ou à emprunter un autre chemin. La diversité des points de vue adoptés par l'étudiant traduit la pluralité des postures qu'il explore dans la construction et la conduite de son intervention. Elle est l'objet de son activité autant que le lieu de la construction de ses capacités, déployée simultanément dans trois lieux que sont le terrain, le séminaire de suivi de stage et le collectif étudiants.

\section{Discussion}

Nous avons présenté dans ce texte un dispositif d'encadrement de stages professionnels, en avons explicité les partis pris et rendu visible la mise en œuvre. Discutons à présent notre expérience à la lumière de contributions intéressées par les modalités d'apprentissage et les dispositifs de formation et de suivi d'interventions.

\subsection{Apprentissage d'un agir professionnel}

Le cheminement des étudiants est accompagné tout au long de l'année par un guidage qui ne transmet pas de solution mais construit et ré-élabore en continu les conditions du développement des gestes de métier des jeunes praticiens.

Les oscillations des étudiants, entre équilibre et déséquilibre, sont les indices de la construction de compétences critiques, appelées aussi des compétences non techniques, c'est-à-dire les compétences à reconnaître en soi ses limites, ses capacités, à avoir une meilleure lucidité (Compétences en Sécurité, les Cahiers de la Sécurité Industrielle de l'ICSI, 2014, p. 6-7). Ces formes de déséquilibre transitoire ont été décrites dans les années 80 par à partir d'une situation expérimentale d'apprentissage de réglage d'oscilloscope (Vermersch, 1978). Son étude montrait qu'il existe dans le temps long un 
plus grand effritement de l'apprentissage chez les étudiants confrontés à un instrument déjà réglé que chez les étudiants confrontés à un instrument déréglé. Dans le champ de la didactique professionnelle, deux modèles d'apprentissage ont pu être comparés (Pastré, 2010). Le premier consiste a demander à l'apprenant d'intégrer un patrimoine et un passé, le second modèle engage un apprentissage à la première personne. L'auteur rappelle que la seule manière d'apprendre à atterrir pour un pilote à un moment donné est de le faire seul.

Le temps des oscillations et des déséquilibres est aussi le temps de l'expression de tensions et de leurs dénouements. À la lecture de travaux en formation des adultes, il constitue probablement une caractéristique de tout dispositif de formation, défini comme un lieu d'interaction, de tensions, de relations voire de transactions entre un dispositif et des dispositions (Lameul et al., 2009).

Dans cette optique, l'autonomie et l'appropriation à la première personne de son intervention par l'apprenti ergonome est une condition pour que l'apprentissage permette le développement du geste professionnel et des compétences critiques qui le fondent. Pour le dire autrement et rappeler notre hypothèse de départ, il nous apparaît que l'exercice précoce et outillé de pouvoirs d'agir des jeunes ergonomes dans leurs interventions leur permet de construire leurs capacités d'agir en ergonomie. La subordination relative des capacités d'agir à l'exercice concret et situé des pouvoirs d'agir est cohérente avec le modèle du sujet du «je peux» qui sous-tend l'orientation pédagogique que nous avons retenue.

\subsection{Développement des capacités collectives de partage d'expérience}

Au-delà des constructions singulières dans la réalisation de leurs interventions, le dispositif de suivi de stage contribue également au développement des capacités collectives de partage d'expérience. Lieu public de confrontation d'idées à l'occasion des séminaires d'encadrement du stage, il permet à chacun de préciser les siennes, de se confronter à des échecs expérimentés ailleurs, d'en étudier les causes, en prévoir les évolutions, de tisser les relations qui seront déterminantes pour la construction de sa propre intervention. Lieu privé d'échanges formels lors de séances de travail ou, plus informel au fil de l'année, il permet une mise à distance de l'expérience en train de se vivre, par le soutien, l'encouragement de ceux qui ont déjà accompli une partie ou la totalité du parcours.

127 Le groupe est ainsi porteur d'une véritable initiation dans l'apprentissage et l'écoute active fournit une matière pour bénéficier de la diversité des situations d'interventions et apprendre à y agir en tissant des liens entre des terrains en apparence très éloignés. Progressivement une bibliothèque des situations d'intervention se construit. La diversité des terrains est également l'occasion d'éprouver l'intérêt d'une vision unifiée de l'homme en activité, au-delà de la traditionnelle dichotomie entre travail et vie quotidienne.

Ce type d'apprentissage s'est développé dans l'aéronautique suite aux nombreux accidents meurtriers dans les années 70-80 sous l'appellation CRM (Crew Resource Management). Les démarches de type CRM ont été déployées dans plusieurs industries à haut risque dont le ferroviaire (Duvenci-Langa, Karsenty, \& Salomé-Martin, 2013). Le principe est basé sur des débats et échanges entre les pairs à partir de leur vécu dans leur 
travail au quotidien, les amenant à réfléchir sur leur fonctionnement et leur place dans le collectif par des techniques d'auto-confrontation croisées.

L'importance de ce collectif de pairs au sein de ce dispositif nous apparait comme complémentaire à d'autres dispositifs (Beaujouan, Coutarel, \& Daniellou, 2013). Ces auteurs s'interrogent sur les apports et limites du récit de professionnels et son impact sur le développement des apprentis ergonomes en formation. Une des principales limites identifiée a trait à la manière dont les apprentis ergonomes perçoivent, interprètent le récit et sont en capacité d'exploiter l'expérience qui leur est livrée.

Au sein du dispositif pédagogique que nous avons présenté la co-habitation des espaces formels et informels permet la transmission d'expériences entre étudiants. Dans cette même lignée, Chaubet (2013) questionne les conditions favorables à une réflexion collective de l'expérience. Outre l'absence de dimension évaluatrice directe dans ces espaces d'échanges, l'auteur rappelle l'importance d'une confrontation concrète aux actions et aux pensées des pairs. À l'aune de ces critères, il nous semble que l'importance octroyée au collectif en formation joue un rôle non négligeable dans le développement des étudiants.

\subsection{Formation au métier}

131 Si on s'attache au dispositif de formation, son ambition puise dans la contribution pionnière du CNAM sous l'impulsion d'A. Wisner que nous avons évoquée en partie initiale de ce texte.

Les difficultés des jeunes ergonomes ont pu être identifiées, les moyens et les ressources du guidage mis au jour. De façon convergente avec nos travaux, les résultats de l'étude de Vézina et Baril (2009) viennent enrichir notre expérience. Les auteurs notent qu'une part importante des difficultés des étudiants tiennent " au comment faire », c'est-à-dire aux interrelations avec leurs interlocuteurs de terrain. En considérant les étapes de la démarche, les phases de préparation du mandat (analyse de la demande) et de prédiagnostic concentrent une part importante de difficultés. Elles tiennent notamment à la nécessité de construire rapidement une compréhension de la situation, l'importance de créer un climat de confiance et de gagner en indépendance pour l'analyse de la demande. Ces difficultés sont proches de celles que nous avons identifiées et, de façon convergente avec notre propre dispositif, le groupe de suivi mis en place dès le début de l'intervention est un outil qui contribue à rendre visible l'intervention et à élargir le cercle des acteurs qui pourraient en être partie prenante.

133 Un point commun se dégage de ces expériences pédagogiques. Il semble que c'est que dans la conduite de leurs interventions que les étudiants sont les plus démunis, cependant que dans la construction de leur intervention, en dépit des difficultés qui peuvent être conséquentes, ils semblent plus outillés dans la formation pour faire y face.

Deux perspectives d'évolution du dispositif se dessinent. La première tient dans la nécessité d'enseignements dédiés à la conduite de l'intervention. C'est une des conclusions de l'étude de Vézina et Baril à laquelle nous souscrivons. Consacrer des enseignements spécifiques à la conduite de l'intervention permettrait de structurer un outillage qui est actuellement mobilisé au fur et à mesure de la progression des interventions (par exemple, la simulation des premières rencontres avec les acteurs de l'entreprise, la construction du groupe de suivi). 
La seconde a trait à la place accordée aux tuteurs en entreprise. Nous avons envisagé trois espaces de construction collective de l'intervention, il serait nécessaire de travailler à une articulation plus ferme entre ces collectifs, en particulier le collectif encadrants-étudiants et le groupe de suivi en entreprise. Une construction plus affirmée permettrait une meilleure lisibilité des orientations de formation, voire une mise en partage contribuant ainsi à progresser dans une définition de l'intervention comme lieu de construction sociale et scientifique.

\section{BIBLIOGRAPHIE}

Barcellini, F., Van Belleghem, L., \& Daniellou, F. (2013). Les projets de conception comme opportunité de développement des activités. In Falzon, P. (Ed), Ergonomie constructive (pp. 192-206). Paris : Presses Universitaires de France.

Barthe, B., Quéinnec, Y., \& Verdier, F.(2004). L'analyse de l'activité de travail en poste de nuit : bilan de 25 ans de recherches et perspectives. Le Travail Humain, 67(1) 41-61.

Bationo-Tillon, A., Lahoual, D., Alexandre, D., Mateev, C., Tichadou, C., \& Decortis, F. (2013). Les activités muséales et les médiations culturelles : de nouveaux terrains d'études et de nouvelles collaborations pour l'ergonomie. Actes $d u 48^{e}$ Congrès de la SELF, Paris.

Beaujouan, J., Coutarel, F., \& Daniellou, F. (2013). L'expérience des autres dans la formation : apports et limites du récit professionnel. Éducation Permanente, 196(1), 25-38.

Béguin, P. (2010). Conduite de projet et fabrication collective du travail, une approche développementale. Document de synthèse en vue de l'obtention de l'Habilitation à Diriger des Recherches, Université de Bordeaux 2, 19 Novembre 2010.

Bjerknes, G., Ehn, P., \& Kyng, M. (1987). Computer an democracy - a scandinavian challenge. Avebury, Alershot.

Carré, P. (2005). L'apprenance, vers un nouveau rapport au savoir. Paris : Dunod.

Carré, P. (2016). L'apprenance, des dispositions aux situations. Éducation Permanente, 207(2), 7-24.

Chaubet, P. (2013). Des conditions favorables à une réflexion collective de l'expérience. Éducation Permanente, 196(1) 53-64.

Clot, Y. (1995). Le travail sans l'homme. Paris : La Découverte.

Compétences en Sécurité (2014). Les cahiers de la Sécurité Industrielle de l'ICSI.

Couillaud, S. (2011). Quand les ergonomes rencontrent les concepteurs de mobiliers de jardin. Mémoire de Master 2 en ergonomie, Université Paris 8, Document non publié.

Curie, J. (2000). Travail, personnalisation et changements sociaux. Archives pour une psychologie de travail. Toulouse : Octarès.

Curie, J., \& Hajjar, V. (1987. Vie au travail, vie hors travail La vie en temps partagé. In C. LevyLeboyer \& J.-C. Sperandio (Eds.) Traité de psychologie du travail, (pp. 174-180). Paris : Presses Universitaires de France. 
Daniellou, F. (1996). (Ed) L'ergonomie en quête de ses principes, débats épistémologiques. Toulouse : Octarès.

Daniellou, F. (2004). L'ergonomie dans la conduite de projets de conception de systèmes de travail. In P. Falzon (Ed), Ergonomie (pp. 359-373). Paris : Presses Universitaires de France.

Daniellou, F. (2006). « Je me demanderais ce que la société attend de nous... ». À propos des positions épistémologiques d'Alain Wisner. Travailler, 15(1), 23-38.

Dessors, D. (2006). « Les stages plein temps », différents enjeux sociaux d'un dispositif pédagogique. Travailler, 1(15), 199-205.

Durrafourg, J. (2004). Alain Wisner et l'histoire de l'ergonomie, le mode de réflexion d'A. Wisner. In A. Durrafourg \& B. Vuillon (Eds), Alain Wisner et les tâches du présent - la bataille du travail réel (pp. 13-26). Toulouse : Octarès.

Duvenci-Langa S., Karsenty, L., \& Salomé-Martin, M. (2013). La prise en compte des facteurs humains dans le développement des compétences en sécurité ferroviaire. 2nd UIC World Congress in Rail Training.

Ehn, P. (1988). Workoriented design of compute artifacts. Stockhom : Arbetslivs centrum.

Folcher, V. (2010). Développement des hommes et des techniques, perspectives de conception pour et dans l'usage. Document de synthèse en vue de l'Habilitation à Diriger des Recherches, 22 Juin 2010, Université Paris 8.

Folcher, V. (2015). Concevoir pour et dans l'usage, la maitrise d'usage en conduite de projet. Revue des Interaction Humaines Médiatisées (RIHM), 15(1) 39-60.

Folcher, V., \& Rabardel, P. (2004a). Hommes artefacts, activités : la perspective instrumentale. In P. Falzon (Ed.), Ergonomie (pp. 251-268). Paris : Presses Universitaires de France.

Folcher, V. \& Rabardel, P. (2004b). Artifacts as design-for-use propositions for design-in-use activity. Invited conference to the symposium "Perception, Communication and Activity". International Congress of Psychology (ICP 2004), 7-13 Août 2004, Beijin : China.

Folcher, V., Zreik, K., Ben Rajeb, S., \& Leclercq, P. (2013). Innovative learning for collaborative design in ergonomics, In R. Stouffs \& S. Sariyildiz (Eds), Education and Research in Computer Aided Architectural Design in Europe, (pp. 605-614). Delft University of Technology.

Gadbois, C. (1975). L'analyse des emprise réciproques de lavie de travail sur lavie hors travail. Bulletin du CERP, XXIII(2) 117-151.

Gadbois, C., \& Quéinnec, Y. (1984). Travail de nuit, rythmes circadiens et régulation des activités. Le Travail Humain, 47 195-226.

Garrigou, A. (1992). Les apports des confrontations d'orientation socio-cognitives au sein des processus de conception participatifs : le rôle de l'ergonomie. Thèse de doctorat en ergonomie, Paris, CNAM.

Garrigou, A., Thibault, J-F., Jackson, M, \& Mascia, F. (2001). Contributions et démarche de l'ergonomie dans les processus de conception. Pistes, 3(2) 1-18.

Gouédard, C., \& Rabardel, P. (2012). Pouvoir d'agir et capacités d'agir : une perspective méthodologique ? Illustration dans le champ de la santé, sécurité et conditions de travail. Pistes, 14, 2-29.

Guenfoud, T. (2015). Prise en charge du diabète de type 1 chez de jeunes enfants lors d'un séjour thérapeutique, entre pouvoir d'agir et autonomie masquée. Mémoire de Master 1 en ergonomie, Université Paris 8, Document non publié. 
Guérin, F. Laville, A., Daniellou, F., Duraffourg, J., \& Kerguelen, A. (2001). Comprendre le travail pour le transformer, la pratique de l'ergonomie. ANACT $2^{\mathrm{e}}$ édition 2007.

Kaptelinin, V (2005). The object of activity. Making sense of the sense maker. Mind, Culture and Activity, 12(1), 4-18.

Lago, D. (2009). La contribution d'Henri Desroche à l'éducation des adultes. De la recherche de la « communauté » à la recherche « compagnonnique » : un modèle d'apprentissage permanent. Doctorat en sciences de l'éducation. Université de Lyon 1.

Lahoual, D. (2012). Analyse des activités domestiques de clients producteurs d'énergie photovoltaïque et recommandations pour la conception. Master 2 d'ergonomie. Université Paris 8.

Lameul, L-G., Jezegou, A., \& Trollat, A.-F. (2009). Articuler dispositifs de formation et dispositions des apprenants. Lyon : Chronique sociale.

Laville, A., \& Volkoff, S. (2004). Vieillissement et travail. In P. Falzon (Ed.), Ergonomie (pp. 145-158). Paris : Presses Universitaires de France.

Malrieu, P. (1979). La crise de personnalisation, ses sources et ses conséquences sociales. Psychologie et Éducation, 3, 1-18.

Martin,C. (2004). L'ergonome dans les projets architecturaux. In P. Falzon (Ed.), Ergonomie (pp. 422-435). Paris : Presses Universitaires de France.

Martin, C., Daniellou, F., \& Escouteloup, J. (1996). La demande qui n'existait pas. Actes du $21^{e}$ congrès de la SELF, Bruxelles, pp. 325-329.

Noulin, M. (2002). Ergonomie. Toulouse : Octarès.

Pastré, P. (2010). La didactique professionnelle. Approche anthropologique du développement chez les adultes. Paris : Presses Universitaires de France

Poret, C. (2015). Concevoir pour le Pouvoir d'Agir Ensemble d'un collectif transverse. Le cas de la relation de service dans le domaine commercial. Thèse de doctorat - Université Paris VIII

Poret, C., Folcher, V., Motté, F., \& Haradji, Y. (2016). Concevoir pour le pouvoir d'agir ensemble au sein des organisations : le cas d'un processus commercial. Activités, 13(2), http:// activites.revues.org/2820

Prunier-Poulmaire, S. (1997) Contraintes et exigences de la tâche : la double détermination du travai posté. Doctorat d'Ergonomie, École Pratique des Hautes Études, EPHE : Paris.

Rabardel, P. (2005). Instrument subjectif et développement du pouvoir d'agir In P. Rabardel \& P. Pastré (Eds.), Modèles du sujet pour la conception. Dialectiques, activités, développement (pp. 11-29). Toulouse : Octarès.

Rabardel, P., Folcher, V., \& Le Jollif, G. (1996). Méthodologie d'analyse de la demande : un outil pour l'enseignement et la pratique. In R. Patesson (Ed.), Intervenir par l'ergonomie : regards, diagnostics et actions de l'ergonomie contemporaine (pp. 284-290). Actes du 21ème congrès de la SELF, Bruxelles.

Rabardel, P., \& Samurçay, R. (2001). Artifact mediated learning, New Challenges to Research on Learning. International Symposium organized by the Center for Activity Theory and developmental Work Research, (pp. 21-23). University of Helsinki.

St Vincent, M., Vezina, N., Bellemare, M., Denys, D., Ledoux, E., \& Imbeau, D. (2011). L'intervention en ergonomie. Multimondes. 
Theureau, J., \& Pinsky, L. (1984). Paradoxe de l'ergonomie de conception et logiciel informatique. Revue des Conditions de Travail, 9, 25-31.

Vermersch, P. (1978). Une problématique théorique en psychologie du travail. Le Travail Humain, 41(2), 265-278.

Vézina, N., \& Baril, R. (2009). Apprendre à intervenir : difficultés rencontrées par de jeunes ergonomes et stratégies. Actes $d u 40^{e}$ congrès de l'Association canadienne d'ergonomie : Penser et agir avec l'ergonomie, Calgary, A.C.E, ISBN 978-0-9732384-8-8.

Vygotky, S.-L. (1985). La méthode instrumentale en psychologie. In B. Schnewly \& J.P Bronckart (Eds.), Vygostky aujourd'hui (pp. 39-48). Delachaux \& Niestlé,

Wallon, H. (1985). Les milieux, les groupes et la psychogenèse de l'enfant. Enfance, numéro spécial : Wallon, psychologie et éducation de l'enfance, 95-104. $7^{\mathrm{e}}$ édition.

Wisner, A. (1990). La méthodologie en ergonomie : d'hier à aujourd'hui. Performances humaines et techniques, 50, 32-39.

\section{NOTES}

1. En particulier les acteurs intégrés au groupe de suivi.

2. Et toute analyse du travail (cf. I.1 Intervenir dans les milieux de travail et de vie).

3. P.A.S.T : Professeur associé des établissements publics d'enseignement relevant du ministère chargé de l'éducation nationale.

4. Notons ici que si la frustration des étudiants est réelle, la possibilité d'assurer des séances d'encadrement collectif exige au plan de la formation de dégager des volumes d'enseignements conséquents pour permettre aux encadrants investis une rémunération et/ou une comptabilisation des heures de travail réalisées.

5. Prolepse : en narratologie, la prolepse ou anticipation est une figure de style par laquelle sont mentionnés des faits qui se produiront bien plus tard dans l'intrigue.

\section{RÉSUMÉS}

L'outillage des apprentis ergonomes pour l'intervention fait partie intégrante de la formation professionnelle dans laquelle les étudiants s'engagent en Master. Notre contribution à un dispositif d'encadrement de stages professionnalisants est basée sur l'hypothèse que c'est dans l'exercice précoce de pouvoirs d'agir sur leurs terrains d'intervention que les jeunes ergonomes développent les capacités d'agir qui fondent leurs gestes de métier. Dans une première partie de l'article, nous présentons nos partis pris formatifs et nous les situons dans un ensemble de contributions d'ergonomie et de psychologie. Nous détaillerons en seconde partie le dispositif de suivi de stage en explicitant son principal objectif, former par la mise en débat des interventions en train de se faire. Nous rendons compte ensuite de sa mise en œuvre en examinant les tensions et relâchements qui rythment la construction des interventions dans une co-activité entre enseignants et étudiants. La dernière partie de l'article est consacrée à une discussion thématique sur les liens entre apprentissage et dispositif de formation. 
Ensuring that young ergonomists are properly equipped for intervention in organizations is an integral part of Master's degree vocational training. Our contribution to a device for the supervision of professional training courses is based on the hypothesis that it is in the early exercise of powers to act within there areas of intervention that young ergonomists develop capacities to act as professionals. In the first part of the article we set out the bases of our training and place them within a set of scientific contributions in the fields of ergonomics and psychology. In the second part we provide a detailed description of the supervision device and clarify its main objective - that of training through discussion of ongoing interventions. We then report on its implementation by highlighting the tensions and easings which give rhythm to the construction of the interventions during coactivity between teachers and students. The final part of the paper is dedicated to a thematic discussion on the links between practice and training.

\section{INDEX}

Mots-clés : intervention ergonomique, sujet capable, capacité et pouvoir d'agir, formation professionnelle

Keywords : ergonomic intervention, capable subject, capacity and power to act, professional training

\section{AUTEURS}

\section{VIVIANE FOLCHER}

Université Paris 8 - CiTU Paragraphe, ComUE Paris Lumières

2 rue de la liberté 93526 Saint Denis cedex

viviane.folcher@univ-paris8.fr

\section{ANNE BATIONO-TILLON}

Université Paris 8 - C3U Paragraphe, ComUE Paris Lumières

2 rue de la liberté 93526 Saint Denis cedex

anne.bationo-tillon@univ-paris8.fr

\section{STELLA DUVENCI-LANGA}

SNCF - Direction Sécurité Système - 20 rue de Rome, 75008 Paris

stella.duvenci-langa@sncf.fr 